\title{
End stage renal disease is associated with development of dementia
}

\author{
Peir-Haur Hung ${ }^{1,2}$, Chih-Ching Yeh ${ }^{3,4}$, Chih-Yen Hsiao ${ }^{1,5}$, Pi-Shan Sung ${ }^{6,7}$, Chih-Hsin \\ Muo $^{8}$, Fung-Chang Sung ${ }^{8,9}$, Kuan-Yu Hung ${ }^{10}$ and Kuen-Jer Tsai ${ }^{6,11}$ \\ ${ }^{1}$ Department of Internal Medicine, Ditmanson Medical Foundation Chia-yi Christian Hospital, Chia-yi, Taiwan \\ ${ }^{2}$ Department of Applied Life Science and Health, Chia-Nan University of Pharmacy and Science, Tainan, Taiwan \\ ${ }^{3}$ School of Public Health, College of Public Health and Nutrition, Taipei Medical University, Taipei, Taiwan \\ ${ }^{4}$ Department of Public Health, China Medical University, Taichung, Taiwan \\ ${ }^{5}$ Department of Hospital and Health Care Administration, Chia-Nan University of Pharmacy and Science, Tainan, Taiwan \\ ${ }^{6}$ Institute of Clinical Medicine, College of Medicine, National Cheng Kung University, Tainan, Taiwan \\ ${ }^{7}$ Department of Neurology, National Cheng Kung University Hospital, Tainan, Taiwan \\ ${ }^{8}$ Management Office for Health Data, China Medical University Hospital, Taichung, Taiwan \\ ${ }^{9}$ Graduate Institute of Clinical Medical Science, School of Medicine, College of Medicine, China Medical University, Taichung, \\ Taiwan \\ ${ }^{10}$ Department of Internal Medicine, National Taiwan University Hospital, Hsin-Chu Branch, Hsin-Chu City, Taiwan \\ ${ }^{11}$ Center of Clinical Medicine, National Cheng Kung University Hospital, College of Medicine, National Cheng Kung University, \\ Tainan, Taiwan
}

Correspondence to: Kuen-Jer Tsai, email: kjtsai@mail.ncku.edu.tw

Keywords: Alzheimer's disease, end stage renal disease, hemodialysis, peritoneal dialysis, dementia, Gerotarget

Received: March 29, 2017 Accepted: October 12, $2017 \quad$ Published: November 15, 2017

Copyright: Hung et al. This is an open-access article distributed under the terms of the Creative Commons Attribution License 3.0 (CC BY 3.0), which permits unrestricted use, distribution, and reproduction in any medium, provided the original author and source are credited.

\section{ABSTRACT}

There is controversy regarding the extent of risk for dementia in patients with end stage renal disease (ESRD) who are undergoing hemodialysis (HD) or peritoneal dialysis (PD). We examined data from Taiwan's Longitudinal Health Insurance Database, and used Cox proportional hazard regression analysis to compare the development of dementia in 72,934 HD and PD patients with 72,934 matched controls from January 1, 1999 to December 31, 2010. The results indicate an increased risk for dementia overall in HD patients (adjusted hazard ratio $[\mathrm{aHR}]=1.64, p<0.0001$ ) and PD patients $(\mathrm{aHR}=\mathbf{2 . 2 1}, p<\mathbf{0 . 0 0 0 1})$. HD and PD patients also had significantly greater aHRs for vascular dementia (VaD) and unspecified dementia (UnD), but only HD patients had a significantly greater aHR for Alzheimer's disease (AD). Further research is needed to confirm whether management of ESRD with PD can reduce the incidence of $A D$, and to identify the mechanisms by which ESRD increases the risk of dementia.

\section{INTRODUCTION}

Dementia is a major cause of death and disability among elderly individuals in the general population [1], and advanced age, cerebrovascular disease, and cardiovascular risk factors (diabetes, hypertension, hyperlipidemia, and smoking) increase the risk for dementia [2]. Recent studies suggest that individuals with end-stage renal disease (ESRD) have a 2-7-fold higher prevalence of cognitive impairment and dementia than the general population [3-5].

The pathogenic mechanisms responsible for the relationship between cognitive decline and kidney dysfunction may be similar to those responsible for relationship between cognitive decline and other cardiovascular disorders, such as atherosclerosis, clinical stroke, silent stroke, oxidative stress, and white matter lesions [6-8]. Other pathological conditions, such as 
neurotoxicity of the uremic state, may also explain the relationship of chronic kidney disease (CKD) with cognitive disorders such as Alzheimer's disease (AD), especially in the absence of obvious cerebrovascular disease [9]. Previous studies of patients with ESRD have determined that several uremia-related factors increase the risk for cognitive impairment, including insufficient dialysis, anemia, and aluminum exposure [10-12], although not all of these findings have been confirmed.

The general objective of our research was to determine whether the association of ESRD with cognitive decline can be explained by medical comorbidities or mode of dialysis. Thus, we used the Longitudinal Health Insurance Database (LHIRD) of Taiwan to determine the risk of dementia in ESRD patients undergoing hemodialysis (HD) or peritoneal dialysis (PD) relative to matched healthy controls, and we also analyzed the relationships of HD and PD with 3 different dementia subtypes.

\section{RESULTS}

After propensity score matching, we identified 72,934 ESRD patients (63,372 undergoing HD and 9,562 undergoing PD) and 72,934 control subjects. Table 1 shows the demographic characteristics and comorbidities of these subjects. The only significant difference was that the HD and PD patients had shorter mean follow-up times than the control subjects (standardized mean difference $(\mathrm{SMD})=0.427$ and $\mathrm{SMD}=0.705$, respectively).

Table 2 shows the crude hazard ratios (HRs) and adjusted HRs (aHRs) for dementia in the different groups during the study period. In the entire study population of 145,868 patients, 9751 patients $(6.68 \%)$ developed dementia, $5009(6.87 \%)$ in the ESRD cohort and 4752 $(6.52 \%)$ in the control cohort. A total of $4706 \mathrm{HD}$ patients (7.43\%) and 303 PD patients (3.17\%) developed dementia. Cox proportional hazard regression analysis indicated the aHR for dementia was 1.64 (95\% confidence interval (CI) $=1.58$ to $1.71 ; p<0.0001)$ in HD patients and $2.21(95 \%$ $\mathrm{CI}=1.87$ to $2.62 ; p<0.0001)$ in PD patients.

Figure 1 shows the dementia-free survival curves for the HD cohort (top) and PD cohort (bottom) relative to propensity score-matched controls. Both comparisons indicate that patients undergoing dialysis had significantly increased risk of dementia.

The multivariate Cox model shows that the risk of dementia increased with age in the HD and PD groups (Table 3). For HD patients, the risk of dementia was significantly greater for females $(\mathrm{aHR}=1.10,95 \% \mathrm{CI}=1.05$ to 1.14$)$, and for those with diabetes mellitus ( $\mathrm{aHR}=1.28,95 \% \mathrm{CI}=1.23$ to 1.34 ), hypertension ( $\mathrm{aHR}=1.23,95 \% \mathrm{CI}=1.13$ to 1.34 ), coronary heart disease $(\mathrm{CHD})(\mathrm{aHR}=1.20,95 \% \mathrm{CI}=1.15$ to 1.26$)$, or heart failure (HF) $(\mathrm{aHR}=1.26,95 \% \mathrm{CI}=1.18$ to 1.33). For PD patients, the risk of dementia was significantly greater for those with diabetes mellitus $(\mathrm{aHR}=1.36,95 \% \mathrm{CI}$ $=1.15$ to 1.61$)$ and $\mathrm{CHD}(\mathrm{aHR}=1.30,95 \% \mathrm{CI}=1.09$ to 1.55$)$.
Table 4 shows the crude HRs and aHRs for development of 3 different subtypes of dementia in the HD and PD cohorts. In comparison with the controls, HD patients were more likely to develop each subtype of dementia (AD: $\mathrm{aHR}=1.30, p=0.01$; vascular dementia $(\mathrm{VaD}): \mathrm{aHR}=1.57$, $p<0.0001$; unspecified dementia (UnD): $\mathrm{aHR}=1.67, p<$ $0.0001)$. However, PD patients were more likely to develop $\mathrm{VaD}(\mathrm{aHR}=2.08, p=0.002)$ and $\mathrm{UnD}(\mathrm{aHR}=2.26, p<$ $0.0001)$, but not $\mathrm{AD}(\mathrm{aHR}=1.77, p=0.14)$.

\section{DISCUSSION}

To our knowledge, the present study is the first nationwide population-based cohort study to compare the incidence rates of $\mathrm{AD}, \mathrm{VaD}$, and $\mathrm{UnD}$ in patients undergoing $\mathrm{HD}$ and $\mathrm{PD}$ with matched controls from the general population. More specifically, we used multivariable regression analysis to retrospectively compare the development of dementia over a 12 year period in ESRD patients undergoing HD or PD with controls who did not have ESRD. The results indicate that ESRD patients undergoing $\mathrm{HD}$ or $\mathrm{PD}$ have a significantly increased risk for dementia. Moreover, our analysis of dementia subtypes indicated that HD and PD patients have increased risk for $\mathrm{VaD}$ and $\mathrm{UnD}$, and that HD patients- but not PD patients- have an increased risk for AD.

Our results clearly indicate an increased risk of dementia in the HD and PD cohorts relative to the ageand sex-matched controls. It is possible that ESRD and dementia share several pathological pathways, and develop simultaneously during aging due to the accumulation of "traditional" vascular risk factors. Alternatively, ESRD may lead to dementia due to "nontraditional" vascular risk factors caused by renal impairment, such as anemia, oxidative stress, chronic inflammation, and uremic toxins [13]. These nontraditional factors are associated with generalized endothelial dysfunction and systemic vascular remodeling $[14,15]$. Moreover, dementia and ESRD may also be similar in that they have certain genetic factors in common and in that each is characterized by the accumulation of certain metabolic toxins [16]. Indeed, a recent meta-analysis of 10 prospective studies showed a significant association of CKD with cognitive impairment [17]. Furthermore, vascular injury and damage from a stroke or white-matter lesion contributes greatly to cognitive decline in AD [18]. Therefore, the findings of the present study provide a basis for investigating the underlying neuropathophysiological mechanisms that link ESRD with the development of dementia.

HD and PD each has its advantages and disadvantages, in that each has different impacts on a patient's physical, psychological, and social health, and each places unique limitations on a patient's lifestyle [19]. The limited available data suggest that the prevalence of cognitive impairment might be lower in PD patients than 
Table 1: Demographic characteristics and comorbidities of the ESRD and control groups

\begin{tabular}{|c|c|c|c|c|c|c|c|c|c|c|}
\hline & \multicolumn{4}{|c|}{ HD } & \multirow{3}{*}{$\begin{array}{l}\text { Standardized } \\
\text { difference }\end{array}$} & \multicolumn{4}{|c|}{ PD } & \multirow{3}{*}{$\begin{array}{c}\text { Standardized } \\
\text { difference }\end{array}$} \\
\hline & \multicolumn{2}{|c|}{$\begin{array}{c}\text { Yes } \\
(N=63372)\end{array}$} & \multicolumn{2}{|c|}{$\begin{array}{c}\text { No } \\
(N=63372)\end{array}$} & & \multicolumn{2}{|c|}{$\begin{array}{c}\text { Yes } \\
(N=9562)\end{array}$} & \multicolumn{2}{|c|}{$\begin{array}{c}\text { No } \\
(N=9562)\end{array}$} & \\
\hline & $n$ & $\%,(\mathrm{SD})$ & $n$ & $\%,(\mathrm{SD})$ & & $n$ & $\%,(\mathrm{SD})$ & $n$ & $\%,(\mathrm{SD})$ & \\
\hline Mean age, years (SD) & 61.2 & $(13.9)$ & 62.2 & $(13.7)$ & 0.069 & 52.9 & $(15.0)$ & 54.0 & $(14.3)$ & 0.072 \\
\hline \multicolumn{11}{|l|}{ Sex } \\
\hline Male & 32389 & 48.9 & 31788 & 50.2 & 0.019 & 4435 & 46.4 & 4414 & 46.2 & 0.004 \\
\hline Female & 30983 & 51.1 & 31584 & 49.8 & 0.019 & 5127 & 53.6 & 5148 & 53.8 & 0.004 \\
\hline \multicolumn{11}{|l|}{ Residence } \\
\hline Urban & 34492 & 54.4 & 34362 & 54.2 & 0.04 & 5988 & 62.6 & 5918 & 61.9 & 0.015 \\
\hline Suburban & 20927 & 33.0 & 20895 & 33.0 & 0.01 & 2760 & 28.9 & 2844 & 29.7 & 0.019 \\
\hline Rural & 7953 & 12.6 & 8115 & 12.8 & 0.01 & 814 & 8.51 & 800 & 8.37 & 0.019 \\
\hline Mean follow-up, years (SD) & 4.24 & $(3.15)$ & 5.65 & $(3.45)$ & 0.427 & 3.23 & $(2.43)$ & 5.25 & $(3.24)$ & 0.705 \\
\hline Coronary heart disease & 24360 & 38.4 & 23400 & 36.9 & 0.031 & 2804 & 29.3 & 2939 & 30.7 & 0.031 \\
\hline Hypertension & 56250 & 88.8 & 56422 & 89.0 & 0.009 & 8507 & 89.0 & 8521 & 89.1 & 0.005 \\
\hline Diabetes & 28271 & 44.6 & 28584 & 45.1 & 0.010 & 3570 & 37.3 & 3720 & 38.9 & 0.032 \\
\hline Atrial fibrillation & 1128 & 1.78 & 1434 & 2.26 & 0.034 & 111 & 1.16 & 96 & 1.00 & 0.015 \\
\hline Heart failure & 7744 & 12.2 & 7077 & 11.2 & 0.034 & 1291 & 13.5 & 1431 & 15.0 & 0.015 \\
\hline Hyperlipidemia & 26851 & 42.4 & 27620 & 43.6 & 0.025 & 4231 & 44.3 & 4303 & 45.0 & 0.015 \\
\hline Mean propensity score (SD) & 0.37 & $(0.22)$ & 0.37 & $(0.22)$ & 0.001 & 0.11 & $(0.10)$ & 0.11 & $(0.10)$ & 0.001 \\
\hline
\end{tabular}

The HD and PD groups were each age- and sex-matched with controls who did not have ESRD

Table 2: Crude and adjusted hazard ratios for development of dementia over 12 years in ESRD patients undergoing HD or PD relative to matched controls

\begin{tabular}{|c|c|c|c|c|c|c|}
\hline & Control group & HD group & $P$ & Control group & PD group & $P$ \\
\hline No. of cases & 4420 & 4706 & & 322 & 303 & \\
\hline Person-year & 358187 & 268868 & & 50205 & 30916 & \\
\hline Incidence rate $\left(10^{-3}\right)$ & 12.34 & 17.50 & & 6.41 & 9.80 & \\
\hline Crude HR $(95 \%$ CI $)$ & 1.00 & $1.40(1.35-1.46)$ & $<0.0001$ & 1.00 & $1.53(1.30-1.80)$ & $<0.0001$ \\
\hline Adjusted HR $(95 \% \mathrm{CI})^{\mathrm{a}}$ & 1.00 & $1.64(1.58-1.71)$ & $<0.0001$ & 1.00 & $2.21(1.87-2.62)$ & $<0.0001$ \\
\hline
\end{tabular}

Adjustments were made for age, sex, residence, diabetes, hypertension, coronary heart disease, heart failure, atrial fibrillation, and hyperlipidemia.

in HD patients [20, 21]. Other studies showed that patients with CKD had a higher prevalence of silent cerebral infarctions and a greater degree of brain atrophy [22], and that these changes were apparently related to the duration of HD [23]. In addition, hemodynamic changes related to $\mathrm{HD}$, including a decline in blood pressure, acute metabolic and fluid changes, and more efficient HD (indicated by $\mathrm{Kt} / \mathrm{V}$ ), are associated with a higher incidence of cognitive dysfunction [24]. However, a previous direct comparison of HD and PD patients indicated no significant difference in the development of ischemic stroke [25], possibly explaining why more dialysis patients experience $\mathrm{VaD}$ than AD [3], as well as our finding that HD and PD patients had a significantly increased risk of $\mathrm{VaD}$ and $\mathrm{UnD}$ (Table 4).
Our results indicate that $\mathrm{PD}$ and HD patients had greater risk for $\mathrm{VaD}$ and $\mathrm{UnD}$ than controls, and that the aHRs for both of these subtypes of dementia were greater for PD patients than HD patients. HD and PD generally differ in efficiency (i.e. removal of uremic toxins) and have different effects on patients' physical, psychological, and social health. Although a rapid fluid and electrolyte shift occurs during HD but not PD, PD can lead to fluid overloading and secondary metabolic disorders from the glucose-based dialysate, and this could contribute to VaD. Moreover, our results showed that ESRD patients undergoing HD -- but not PD -- had a greater risk for AD. An imbalance between the production and clearance of $\beta$-Amyloid peptide $(A \beta)$ in the central nervous system 
causes $A \beta$ accumulation, and this may be the most important factor in the pathogenesis of AD [26]. A recent study found that serum $A \beta$ levels were significantly higher in CKD patients who had never received dialysis than those who were receiving $\mathrm{PD}$, and that ESRD patients undergoing PD and non-ESRD controls had comparable serum $A \beta$ levels [27]. Other research indicated that HD only reduced serum $A \beta$ levels by about 30\% [28]. These findings suggest that the kidney has a role in the peripheral clearance of $\mathrm{A} \beta$, and that $\mathrm{PD}$ might lower serum levels of $A \beta$. Future studies of AD in ESRD patients should consider alternative methods to increase the peripheral clearance of $\mathrm{A} \beta$, and should further investigate this potentially important risk factor.

The strengths of this study are that it was a population-based, nationwide study that examined all validated cases of dementia which occurred in patients with ESRD over a 12-year period. All comorbidities and medical interventions were accurately recorded due to the national health insurance policy of Taiwan. However, the current study also has some limitations. First, we did not have measurements of inflammatory markers, direct measures of kidney function, nor measures of nutritional status. Although we adjusted for common health conditions in the multivariate analysis, there is a possibility that certain subclinical diseases may also have contributed to the observed cognitive decline in ESRD patients. Second, we relied exclusively on claims data, and this may have contributed to a bias in disease classification. In particular, doctors usually exclude reversible causes of dementia before making a diagnosis; however, because detailed hematological data were not available, we could not adjust for some potentially confounding factors for cognitive dysfunction in our population. Due to the limitations of the

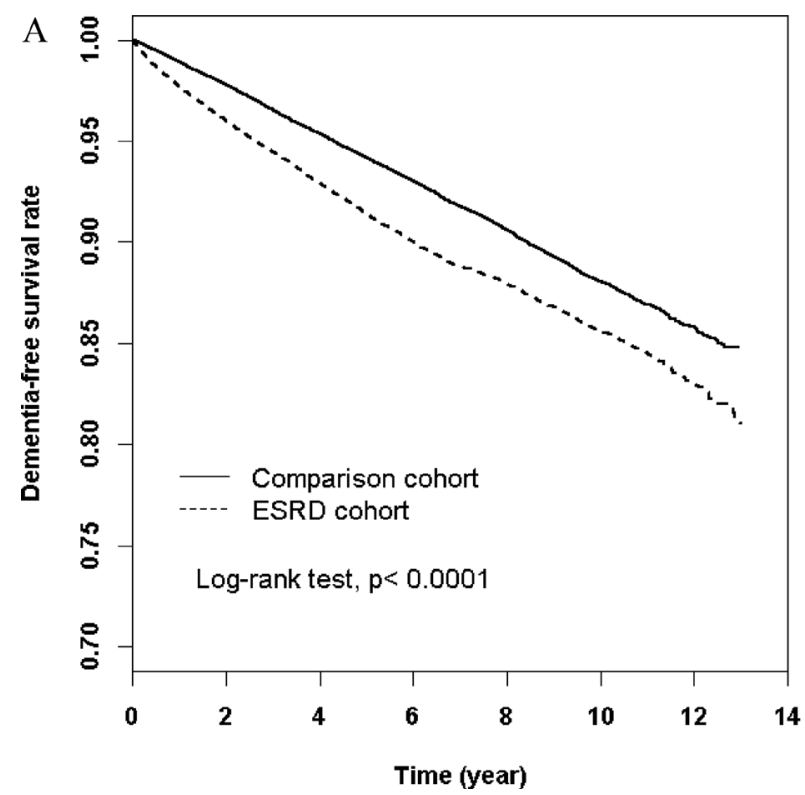

retrospective design of the current study, we are currently developing a detailed mechanism-based study of this topic by use of a prospective cohort design.

In conclusion, our study indicates that patients with ESRD who are undergoing HD or PD have a higher incidence of dementia than those without ESRD. Moreover, ESRD patients undergoing HD - but not PD - have a greater risk of AD than those without ESRD. Further research in this area is needed to identify the pathogenic mechanism(s) that connect ESRD with dementia, and to examine whether management of ESRD with PD rather than HD can effectively reduce the incidence of $\mathrm{AD}$ in these patients.

\section{MATERIALS AND METHODS}

\section{Data collection}

A universal National Health Insurance (NHI) programme was implemented in Taiwan during March 1995, and enrolled $96 \%$ of the total population at that time [29]. By the end of 1996, the Bureau of NHI (BNHI) had contracts with $97 \%$ of all Taiwanese hospitals and clinics to join the NHI system [30]. The BNHI accumulates all administrative and claims data for Taiwan, and the National Health Research Institute (NHRI) collaborated with the BNHI to establish an NHI research database. The NHRI safeguards the privacy and confidentiality of all beneficiaries, and provides health insurance data for research purposes only after obtaining ethical approval. To ensure the accuracy of the claims files, the BNHI conducts expert reviews on random samples for every 50-100 ambulatory and inpatient claims on a quarterly basis. False diagnoses can lead to imposition of severe penalties for the hospitals [31].

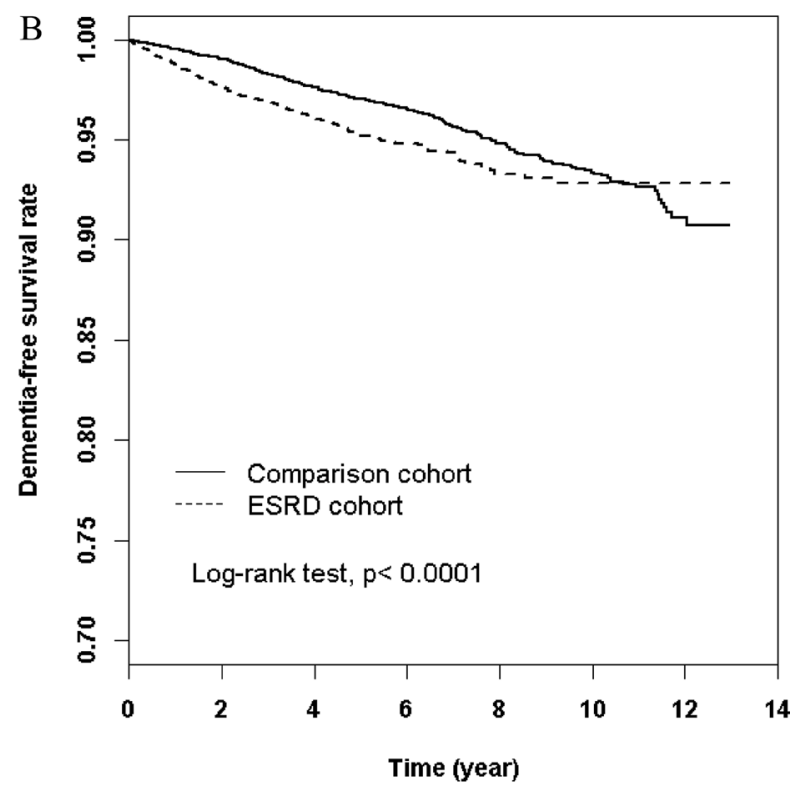

Figure 1: Dementia-free survival rate of ESRD patients. Undergoing HD (A) and PD (B) relative to age-and sex-matched controls. 
Table 3: Multivariate Cox proportional hazards model for development of dementia over 12 years in ESRD patients undergoing HD or PD relative to matched controls

\begin{tabular}{|c|c|c|c|c|}
\hline & HD group & & PD group & \\
\hline & aHR (95\% CI) & $P$ & aHR (95\% CI) & $P$ \\
\hline \multicolumn{5}{|l|}{ Age, years } \\
\hline $18-44$ & 1.00 & & 1.00 & \\
\hline $45-64$ & $6.62(5.29-8.29)$ & $<0.0001$ & $7.02(3.97-12.4)$ & $<0.0001$ \\
\hline$\geq 65$ & $36.3(29.1-45.3)$ & $<0.0001$ & $57.8(32.9-101)$ & $<0.0001$ \\
\hline \multicolumn{5}{|l|}{ Sex } \\
\hline Male & 1.00 & & 1.00 & \\
\hline Female & $1.10(1.05-1.14)$ & $<0.0001$ & $1.13(0.96-1.33)$ & 0.14 \\
\hline \multicolumn{5}{|l|}{ Residence } \\
\hline Urban & $1.03(0.98-1.07)$ & 0.27 & $1.04(0.87-1.24)$ & 0.70 \\
\hline Suburban & 1.00 & & 1.00 & \\
\hline Rural & $1.02(0.96-1.09)$ & 0.53 & $1.06(0.80-1.41)$ & 0.69 \\
\hline HD & $1.64(1.58-1.71)$ & $<0.0001$ & -- & \\
\hline PD & -- & & $2.21(1.87-2.62)$ & $<0.0001$ \\
\hline Diabetes & $1.28(1.23-1.34)$ & $<0.0001$ & $1.36(1.15-1.61)$ & 0.0003 \\
\hline Hypertension & $1.23(1.13-1.34)$ & $<0.0001$ & $1.02(0.72-1.43)$ & 0.93 \\
\hline Coronary heart disease & $1.20(1.15-1.26)$ & $<0.0001$ & $1.30(1.09-1.55)$ & 0.003 \\
\hline Atrial fibrillation & $1.10(0.97-1.24)$ & 0.15 & $0.55(0.27-1.12)$ & 0.20 \\
\hline Heart failure & $1.26(1.18-1.33)$ & $<0.0001$ & $1.17(0.97-1.42)$ & 0.11 \\
\hline Hyperlipidemia & $0.98(0.94-1.02)$ & 0.37 & $1.12(0.94-1.32)$ & 0.20 \\
\hline
\end{tabular}

\section{Study population}

We selected all patients from the NHI database who had ESRD and began maintenance HD or PD between 1 January 1999 and 31 December 2010, and who survived more than 90 days of renal replacement therapy (RRT) $(n=72,934)$. We excluded individuals younger than 18 years of age, because they had no appreciable risk of dementia. All HD and PD patients had catastrophic illness registration cards for treatment of ESRD (International Classification of Diseases, 9th revision, Clinical Modification [ICD-9-CM] code 585). In Taiwan, patients with ESRD can apply to the BNHI for catastrophic illness registration cards, so they have no copayments for longterm RRT. We excluded patients diagnosed with ESRD before the index date, those whose age or sex were not recorded, and those with any type of stroke (codes 430-438) before or within 90 days of the index date for ambulatory care (date of initiating dialysis). A total of 63,372 incident HD patients and 9,562 incident PD patients were identified during the study period. During the 12 months before the index date for ambulatory care, we obtained data on the following potential confounders (all of which are documented risk factors for dementia): hypertension (codes 401-405), diabetes (code 250), hyperlipidemia (code 272), CHD (codes 410-414 or 429.2), atrial fibrillation (AF) (code 427.31), and HF (codes 428).

Control subjects were randomly selected from the remaining subjects in the database using the same exclusion criteria, with exclusion of subjects with incident dementia prior to the index clinic visit. The control group consisted of randomly selected age- and sex-matched individuals who had no histories of ESRD or CKD. Participants with no CKD, but with documented hypertension, diabetes, hyperlipidaemia, $\mathrm{CHD}$, AF, or $\mathrm{HF}$, were included in the control group. The control-to-patient ratio was $1: 1$. We used propensity score matching to reduce selection bias resulting from nonrandom assignment. The age of each study subject was calculated as the time between the index date and the date of birth. Each patient was individually tracked from the index date for use of healthcare facilities to identify those who subsequently suffered from incident dementia (codes 290.0 to 290.4, 294.1, and 331.0 to 331.2), or until December 31, 2011.

\section{Statistical analysis}

The primary endpoint was receipt of an ambulatory care visit or hospitalization for any type of dementia. The 
Table 4: Crude and adjusted hazard ratios for development of different dementia subtypes over 12 years in ESRD patients undergoing HD or PD relative to matched controls

\begin{tabular}{|c|c|c|c|c|c|c|}
\hline & Control group & HD group & $\boldsymbol{P}$ & Control group & PD group & $P$ \\
\hline \multicolumn{7}{|l|}{ Alzheimer's disease } \\
\hline No. of cases & 202 & 168 & & 19 & 13 & \\
\hline Crude HR (95\% CI) & 1.00 & $1.11(0.90-1.36)$ & 0.34 & 1.00 & $1.23(0.60-2.53)$ & 0.58 \\
\hline Adjusted HR $(95 \% \mathrm{CI})^{\mathrm{a}}$ & 1.00 & $1.30(1.06-1.60)$ & 0.01 & 1.00 & $1.77(0.83-3.80)$ & 0.14 \\
\hline \multicolumn{7}{|l|}{ Vascular dementia } \\
\hline No. of cases & 559 & 567 & & 48 & 41 & \\
\hline Crude HR (95\% CI) & 1.00 & $1.33(1.18-1.50)$ & $<0.0001$ & 1.00 & $1.40(0.92-2.15)$ & 0.12 \\
\hline Adjusted HR $(95 \% \text { CI })^{a}$ & 1.00 & $1.57(1.39-1.77)$ & $<0.0001$ & 1.00 & $2.08(1.32-3.28)$ & 0.002 \\
\hline \multicolumn{7}{|l|}{ Unspecified dementia } \\
\hline No. of cases & 3659 & 3971 & & 255 & 249 & \\
\hline Crude HR $(95 \%$ CI $)$ & 1.00 & $1.43(1.37-1.50)$ & $<0.0001$ & 1.00 & $1.58(1.32-1.88)$ & $<0.0001$ \\
\hline Adjusted HR $(95 \% \text { CI })^{\mathrm{a}}$ & 1.00 & $1.67(1.60-1.75)$ & $<0.0001$ & 1.00 & $2.26(1.87-2.73)$ & $<0.0001$ \\
\hline
\end{tabular}

Adjustments were made for significant variables from Table 1 (patient age, gender, urbanization level, diabetes, hypertension, coronary heart disease, heart failure, atrial fibrillation, and hyperlipidemia).

covariates were age, gender, region of residence (urban, suburban, or rural), and selected baseline comorbidities (hypertension, diabetes, hyperlipidemia, CHD, AF, and HF) [32-34]. Categorical variables are expressed as frequencies and percentages, and continuous variables as means and standard deviation (SD). We considered the above comorbidities only if the condition occurred in an inpatient setting, or if there were 2 or more ambulatory care claims recorded 1 year before or after the index ambulatory care visit. The baseline characteristics of the $\mathrm{HD}, \mathrm{PD}$, and control groups were compared using the $\mathrm{SMD}$, calculated as the difference in means or proportions of a variable divided by a pooled estimate of the standard deviation of the variable [35]. This measure is not influenced by sample size, and is useful for comparison of cohorts in large observational studies. An SMD value of 0.1 or less indicates a negligible difference between groups [35].

Cox proportional hazard regression analysis was used to calculate HRs and 95\% CI between ESRD (HD or PD) and dementia. The aHRs were obtained by correcting for demographic characteristics and comorbidities in multivariate Cox regression models. The dementia-free survival rates were estimated by the Kaplan-Meier method, and the log-rank test was used to calculate the significance of differences. We also examined the relationship between ESRD (HD or PD) and different dementia subtypes: AD (code 331.0), VaD (code 290.4), and UnD (codes 290.0 to 290.3, 294.1, and 331.1 to 331.2) [36]. All data analyses were conducted using SAS (ver. 9.3, SAS Institute, Cary, NC) for Windows, and the significance level was 0.05 in a two-sided test.

\section{Abbreviations}

A $\beta, \beta$-Amyloid peptide; AD, Alzheimer's disease; AF, atrial fibrillation; aHR, adjusted hazard ratio; $\mathrm{BNHI}$, Bureau of NHI; CHD, coronary heart disease; CKD, chronic kidney disease; ESRD, end stage renal disease; HD, hemodialysis; $\mathrm{HF}$, heart failure; HR, hazard ratio; NHI, National Health Insurance; $\mathrm{PD}$, peritoneal dialysis; $\mathrm{SD}$, standard deviation; $\mathrm{SMD}$, standardized mean difference; VaD, vascular dementia; UnD, unspecified dementia.

\section{Author contributions}

K.J.T., P.H.H., C.C.Y., and K.Y.H. designed the research; C.C.Y., C.Y.H., P.S.S., F.C.S., and C.H.M. analyzed the data; and K.J.T. and P.H.H. wrote the first draft of the paper. All authors participated in revision of the paper.

\section{ACKNOWLEDGMENTS}

The authors would also like to acknowledge the support of Dr. Tsung-Hsien Chen in preparation of this manuscript.

\section{CONFLICTS OF INTEREST}

All authors declare no conflicts of interest.

\section{FUNDING}

This study was supported in part by the National Cheng Kung University, Taiwan Ministry of Health and 
Welfare Clinical Trial and Research Center of Excellence (MOHW105-TDU-B-212-133019), China Medical University Hospital, Academia Sinica Taiwan Biobank Stroke Biosignature Project (BM10501010037), NRPB Stroke Clinical Trial Consortium (MOST 104-2325-B039-005), Tseng-Lien Lin Foundation (Taichung, Taiwan), Taiwan Brain Disease Foundation (Taipei, Taiwan), and Katsuzo and Kiyo Aoshima Memorial Funds (Japan).

\section{REFERENCES}

1. Prohovnik I, Post J, Uribarri J, Lee H, Sandu O, Langhoff E. Cerebrovascular effects of hemodialysis in chronic kidney disease. J Cereb Blood Flow Metab. 2007; 27:1861-69.

2. Knopman D, Boland LL, Mosley T, Howard G, Liao D, Szklo M, McGovern P, Folsom AR; Atherosclerosis Risk in Communities (ARIC) Study Investigators. Cardiovascular risk factors and cognitive decline in middle-aged adults. Neurology. 2001; 56:42-48.

3. Fukunishi I, Kitaoka T, Shirai T, Kino K, Kanematsu E, Sato Y. Psychiatric disorders among patients undergoing hemodialysis therapy. Nephron. 2002; 91:344-47.

4. Kurella M, Chertow GM, Luan J, Yaffe K. Cognitive impairment in chronic kidney disease. J Am Geriatr Soc. 2004; 52:1863-69.

5. Sehgal AR, Grey SF, DeOreo PB, Whitehouse PJ. Prevalence, recognition, and implications of mental impairment among hemodialysis patients. Am J Kidney Dis. 1997; 30:41-49.

6. Kurella M, Chertow GM, Fried LF, Cummings SR, Harris T, Simonsick E, Satterfield S, Ayonayon H, Yaffe K. Chronic kidney disease and cognitive impairment in the elderly:the health, aging, and body composition study. J Am Soc Nephrol. 2005; 16:2127-33.

7. Murray AM. Cognitive impairment in the aging dialysis and chronic kidney disease populations: an occult burden. Adv Chronic Kidney Dis. 2008; 15:123-32.

8. Seliger SL, Longstreth WT. Lessons about brain vascular disease from another pulsating organ, the kidney. Stroke. 2008; 39:5-6.

9. Seifter JL, Samuels MA. Uremic encephalopathy and other brain disorders associated with renal failure. Semin Neurol. 2011; 31:139-43.

10. Grimm G, Stockenhuber F, Schneeweiss B, Madl C, Zeitlhofer J, Schneider B. Improvement of brain function in hemodialysis patients treated with erythropoietin. Kidney Int. 1990; 38:480-86.

11. Rozas VV, Port FK, Rutt WM. Progressive dialysis encephalopathy from dialysate aluminum. Arch Intern Med. 1978; 138:1375-77.

12. Teschan PE, Bourne JR, Reed RB, Ward JW. Electrophysiological and neurobehavioral responses to therapy: the National Cooperative Dialysis Study. Kidney Int Suppl. 1983; 13:S58-S65.
13. Bugnicourt JM, Godefroy O, Chillon JM, Choukroun G, Massy ZA. Cognitive disorders and dementia in CKD: the neglected kidney-brain axis. J Am Soc Nephrol. 2013; 24:353-63.

14. Mogi M, Horiuchi M. Clinical interaction between brain and kidney in small vessel disease. Cardiol Res Pract. 2011; 2011:306189.

15. Schiffrin EL, Lipman ML, Mann JF. Chronic kidney disease: effects on the cardiovascular system. Circulation. 2007; 116:85-97.

16. Murray AM. The brain and the kidney connection: A model of accelerated vascular cognitive impairment. Neurology. 2009; 73:916-17.

17. Etgen T, Chonchol M, Förstl H, Sander D. Chronic kidney disease and cognitive impairment: a systematic review and meta-analysis. Am J Nephrol. 2012; 35:474-82.

18. O’Brien JT, Erkinjuntti T, Reisberg B, Roman G, Sawada T, Pantoni L, Bowler JV, Ballard C, DeCarli C, Gorelick PB, Rockwood K, Burns A, Gauthier S, et al. Vascular cognitive impairment. Lancet Neurol. 2003; 2:89-98.

19. Lindqvist R, Carlsson M, Sjoden PO. Coping strategies and health-related quality of life among spouses of continuous ambulatory peritoneal dialysis, haemodialysis, and transplant patients. J Adv Nurs. 2000; 31:1398-408.

20. Buoncristiani U, Alberti A, Gubbiotti G, Mazzotta G, Gallai V, Quintaliani G, Gaburri M. Better preservation of cognitive faculty in continuous ambulatory peritoneal dialysis. Perit Dial Int. 1993; 13:S202-05.

21. Wolcott DL, Wellisch DK, Marsh JT, Schaeffer J, Landsverk J, Nissenson AR. Relationship of dialysis modality and other factors to cognitive function in chronic dialysis patients. Am J Kidney Dis. 1988; 12:275-84.

22. Pereira AA, Weiner DE, Scott T, Sarnak MJ. Cognitive function in dialysis patients. Am J Kidney Dis. 2005; 45:448-62.

23. Yoshimitsu T, Hirakata H, Fujii K, Kanai H, Hirakata E, Higashi H, Kubo M, Tanaka H, Shinozaki M, Katafuchi R, Yokomizo Y, Oh Y, Tomooka S, et al. Cerebral ischemia as a causative mechanism for rapid progression of brain atrophy in chronic hemodialysis patients. Clin Nephrol. 2000; 53:445-51.

24. Murray AM, Tupper DE, Knopman DS, Gilbertson DT, Pederson SL, Li S, Smith GE, Hochhalter AK, Collins AJ, Kane RL. Cognitive impairment in hemodialysis patients is common. Neurology. 2006; 67:216-23.

25. Wang HH, Hung SY, Sung JM, Hung KY, Wang JD. Risk of stroke in long-term dialysis patients compared with the general population. Am J Kidney Dis. 2014; 63:604-11.

26. Querfurth HW, LaFerla FM. Alzheimer's disease. N Engl J Med. 2010; 362:329-44.

27. Liu YH, Xiang Y, Wang YR, Jiao SS, Wang QH, Bu XL, Zhu C, Yao XQ, Giunta B, Tan J, Zhou HD, Wang YJ. Association between serum amyloid-Beta and renal functions: implications for roles of kidney in amyloid-Beta clearance. Mol Neurobiol. 2015; 52:115-19. 
28. Kawaguchi K, Kitaguchi N, Nakai S, Murakami K, Asakura K, Mutoh T, Fujita Y, Sugiyama S. Novel therapeutic approach for Alzheimer's disease by removing amyloid beta protein from the brain with an extracorporeal removal system. J Artif Organs. 2010; 13:31-37.

29. Lu JF, Hsiao WC. Does universal health insurance make health care unaffordable? Lessons from Taiwan. Health Aff (Millwood). 2003; 22:77-88.

30. Chiang TL. Taiwan's 1995 health care reform. Health Policy. 1997; 39:225-39.

31. Tseng $\mathrm{CH}$. Mortality and causes of death in a national sample of diabetic patients in Taiwan. Diabetes Care. 2004; 27:1605-09.

32. Almeida OP, Garrido GJ, Beer C, Lautenschlager NT, Arnolda L, Flicker L. Cognitive and brain changes associated with ischaemic heart disease and heart failure. Eur Heart J. 2012; 33:1769-76.

33. Chiang CJ, Yip PK, Wu SC, Lu CS, Liou CW, Liu HC, Liu CK, Chu CH, Hwang CS, Sung SF, Hsu YD, Chen CC,
Liu SI, et al. Midlife risk factors for subtypes of dementia: a nested case-control study in Taiwan. Am J Geriatr Psychiatry. 2007; 15:762-71.

34. Mizrahi EH, Waitzman A, Arad M, Adunsky A. Atrial fibrillation predicts cognitive impairment in patients with ischemic stroke. Am J Alzheimers Dis Other Demen. 2011; 26:623-26.

35. Mamdani M, Sykora K, Li P, Normand SL, Streiner DL, Austin PC, Rochon PA, Anderson GM. Reader's guide to critical appraisal of cohort studies: 2. Assessing potential for confounding. BMJ. 2005; 330:960-62.

36. Wang HK, Lin SH, Sung PS, Wu MH, Hung KW, Wang LC, Huang CY, Lu K, Chen HJ, Tsai KJ. Population based study on patients with traumatic brain injury suggests increased risk of dementia. J Neurol Neurosurg Psychiatry. 2012; 83:1080-85. 